ESDA2006-95625

\title{
NONLINEAR STOCHASTIC CONTROLLERS FOR SEMIACTIVE AND REGENERATIVE STRUCTURAL SYSTEMS, WITH GUARANTEED QUADRATIC PERFORMANCE MARGINS
}

\author{
Jeffrey T. Scruggs \\ Alexandros A. Taflanidis* \\ Wilfred D. Iwan \\ California Institute of Technology, Division of Engineering \& Applied Science \\ 1200 E California Blvd, MC 104-44/ Pasadena, CA 91125 \\ *E-mail: taflanid@caltech.edu, Tel: +01 626-395-4648, Fax:+01 626-395-2719
}

\begin{abstract}
In many applications of vibration control, the circumstances of the application impose constraints on the energy available for the actuation of control forces. Semiactive dampers (i.e., viscous dampers with controllable coefficients) constitute the simplest example of such actuation in structural control applications. Regenerative Force Actuation (RFA) networks are an extension of semiactive devices, in which mechanical energy is first converted to electrical energy, which is then dissipated in a controllable resistive network. A fairly general class of semiactive and regenerative systems can be characterized by a differential equation which is bilinear (i.e., linear in state, linear in control input, but nonlinear in both). This paper presents a general approach to bilinear feedback control system design for semiactive and regenerative systems, which is analytically guaranteed to out-perform optimal linear viscous damping in stationary stochastic response, under the familiar Quadratic Gaussian performance measure. The design for full-state feedback and for the more practical case of noisecorrupted and incomplete measurements (i.e., output feedback) are separately discussed. Variants of the theory are shown to exist for other quadratic performance measures, including risksensitive and multi-objective frameworks. An illustrative application to civil engineering is presented.
\end{abstract}

\section{INTRODUCTION}

Semiactive devices for structural control systems have been studied extensively within the context of automotive, aerospace, and civil applications since the 1970's [1-3]. Their main attraction is that their control forces are inherently dissipative, and the energy required for their operation is only that required for the sensory and intelligence systems.
Consequently, they are highly efficient and much more reliable than active systems. Conventional semiactive systems use mechanical forcing devices such as variable-orifice dampers, controllable-friction devices, and switching systems. These devices allow the rate of energy dissipation to be regulated in real-time, based on sensory feedback measurements. Through proper feedback design, such control can often improve the system response over that attainable with purely passive devices.

Some semiactive devices have been proposed which dissipate energy electrically, using a transducer (such as an electric motor) to facilitate electromechanical energy conversion, and using controllable circuitry to regulate dissipation. A simple schematic for this approach, which is addressed in detail in [4] for an automotive application and in [5] for a civil application, is illustrated in Fig. 1a. From a mechanical point of view, such a system is equivalent to a system of continuously-variable dampers.

The switching networks in Fig. 1a are realized using transistors which open and close different circuit paths to raise and lower the dissipation rate. As with mechanical switches, this mode of operation is highly efficient, requiring orders of magnitude less power than that which is transmitted through the network. As such, this mode of operation is similar to many other semiactive systems. However, it has a unique advantage in that if two or more devices are used to control a structure, their associated electronics may be connected such that electrical power can be transmitted from one actuator to another. Additionally, energy removed from the structure may be stored and reused.

Such regenerative systems of forcing devices, called Regenerative Force Actuation (RFA) networks, have emerged 
in several areas of mechatronics research, with investigations beginning in the automotive suspensions area in the mid-1990's [6-8]. An example of an RFA network is shown in Fig. Ib, which illustrates their two basic advantages. Because the electrical network is global among all actuators, it is possible to transmit energy between remote locations in a structure, in addition to providing dissipation. It is also possible to interface the structural system with a remote energy storage device (e.g., a flywheel), which allows for the energy removed from the main mechanical system to be stored and reused later.
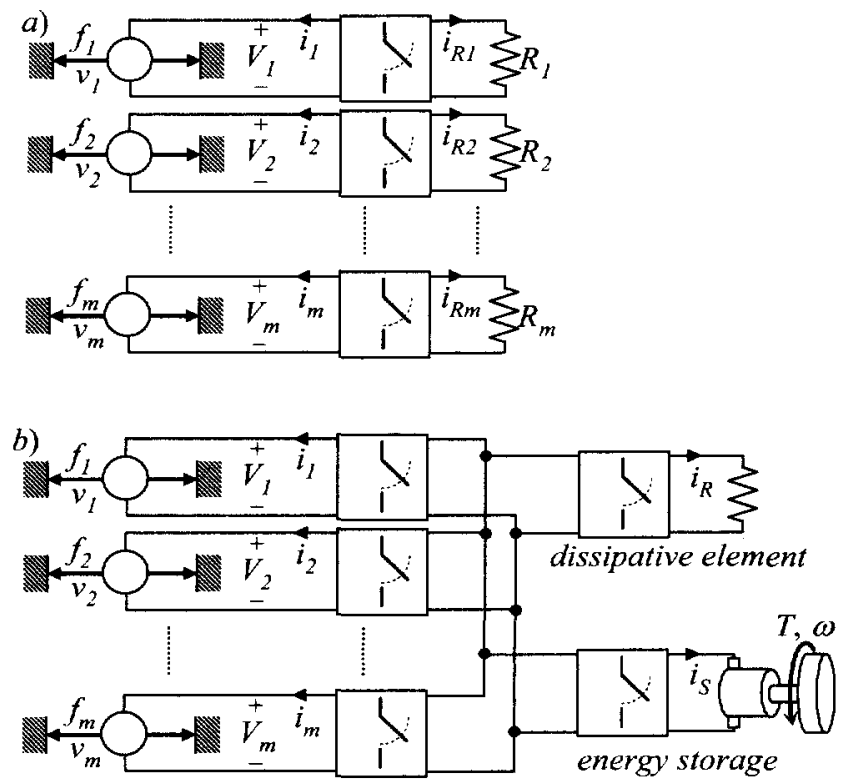

Figure 1: Electromechanical realizations of semiactive (a) \& regenerative (b) actuation

In considering the application of such advanced actuation technology for structural control, the most fundamental question is whether the versatility afforded by the technology justifies the increased associated cost and maintenance issues, beyond those of simpler passive systems. For many advanced actuation technologies such as semiactive devices and RFA networks, the answer to this question has as much to do with the feedback control law as it does with the physical limiatations of the device hardware.

Yet most of the research effort in semiactive and regenerative actuation has been devoted to device design. Meanwhile, the feedback controller design techniques which have been proposed have been mostly heuristic; methods such as hysteretic, dissipation-based, and energy-based approaches. These techniques have gained acceptance in part because they are natural extensions of passive structural response control techniques. However, they do not lead to controllers which explicitly optimize meaningful global measures of dynamic structural performance, such as drifts, accelerations, etc. Although several ad-hoc control design methods (the most popular of which is "clipped-optimal" control [2]) have been proposed which do incorporate such performance measures, they do not in general guarantee any bound on the level of performance actually attained. For structural control applications with few actuators and one dominant mode, such approaches are often sufficient to arrive, after a few design iterations, at a satisfactory controller. However, for systems with many actuators, irregular geometry, or complex dynamics, a more systematic design approach may be desirable.

The methods discussed in the present study are suboptimal Quadratic-Gaussian (QG) controllers; i.e., they do not yield controllers which attain the optimal level of QG performance in stationary response. However, they do produce controllers with easily-computable upper bounds on performance. Furthermore, they are analytically guaranteed to yield superior performance, in comparison to the case where the actuators are replaced with optimized viscous dampers. Investigations of semiactive controllers with a guaranteed performance bound were discussed for a single-device suspension system with full-state feedback by [9], and the research reported in the present analysis can be viewed as an extension of the "steepest descent" control algorithm proposed there.

The design of fullstate feedback and for the more practical case of noise-corrupted and incomplete measurements (i.e., output feedback) are separately discussed. In the latter case both the actuator forces and velocities are assumed to be measured in the absence of noise, and are available as feedback signals. This assumption is reasonable for many control applications of semiactive or regenerative devices.

The potential appeal of the controllers proposed here, which will be called Performance Guaranteed Controllers (PGCs), is that they are comparatively simple control laws. Indeed, they are structurally similar to clipped-optimal controllers, but with different formulas for the assignation of the parameters. Thus, the approach retains practicality for systems with many degrees of freedom and many actuators.

Much of the research reported in this paper is addressed in greater detail in two forthcoming journal papers $[10,11]$. Consequently, in the interest of brevity, the theorems and lemmas are here presented without proof, and an attempt has been made to avoid theoretical nuance.

\section{NOMENCLATURE}

For a vector $\mathbf{q}$ and matrix $\mathbf{Q}$, metrics $\|\mathbf{q}\|_{2}$ and $\|\mathbf{q}\|_{\infty}$ refer to the Euclidean and infinity norms, and $\|\mathbf{Q}\|_{2}$ refers to the maximum singular value. Index notations $q_{i}$ and $Q_{i j}$ will be used to refer to component $i$ of vector $\mathbf{q}$, and component $\{i, j\}$ of matrix $\mathbf{Q}$, respectively.

For a time-valued vector function $\mathbf{q}(t) \in \mathfrak{R}^{p} \times[0, T], \mathbf{q}\left[t_{1}, t_{2}\right]$ is the set $\left\{\mathbf{q}(t): t \in\left[t_{1}, t_{2}\right]\right\}, p(\mathbf{q}(t) \mid \Theta)$ is the probability density function of $\mathbf{q}(t)$ given information $\Theta$, and $E\{\mathbf{q}(t) \mid \Theta\}$ is the associated expectation. 
The notation $\operatorname{argmin}\{\cdot\}$ implies the minimizing argument, and $\operatorname{tr}\{\cdot\}$ is the trace operator. $\{\mathbf{Q}\}_{i j}$ and $Q_{i j}$ both denote element $\{i, j\}$ of matrix $\mathbf{Q}$.

The most frequently-used symbols used in this paper are listed below:

$\begin{array}{ll}\mathbf{f} & \text { Actuator foce vector } \\ \mathbf{v} & \text { Actuator velocity vector } \\ \mathbf{u} & \text { Normalized actuator force; } \mathbf{u}=\mathbf{C}_{e}^{-1 / 2} \mathbf{f} \\ \mathbf{s} & \text { Normalized actuator velocity; } \mathbf{s}=\mathbf{C}_{e}^{1 / 2} \mathbf{v} \\ \mathbf{x} & \text { System state vector } \\ \hat{\mathbf{x}} & \text { Unbiased estimate of } \mathbf{x} \text { (for output feedback) } \\ \mathbf{a} & \text { White noise input in the dynamical system } \\ \mathbf{n} & \text { Sensor noise in the measurements } \\ \mathbf{y} & \text { Vector of performance quantities } \\ \mathbf{z} & \text { Measurement vector (for output feedback) } \\ \mathbf{Z}(t) & \text { Time-varying bilinear control input } \\ \mathbf{Z}_{0} & \text { Denotes time-invariant } \mathbf{Z}(t)=\mathbf{Z}_{0}, \forall t \\ c_{e i} & \text { Maximum viscosity of actuator } i \\ \mathbf{C}_{e} & \text { Diagonal matrix with } C_{e i i}=c_{e i} \\ \mathbf{A}, \mathbf{B}_{u}, \mathbf{B}_{a} & \text { Coefficients in state-space diff. eq. } \\ \mathbf{C}_{y}, \mathbf{D}_{y u} & \text { Coefficients relating } \mathbf{x} \text { and } \mathbf{u} \text { to } \mathbf{y} \\ \mathbf{C}_{z}, \mathbf{D}_{z u}, \mathbf{D}_{z n} & \text { Coefficients relating } \mathbf{x}, \mathbf{u}, \text { and } \mathbf{n} \text { to } \mathbf{z} \\ J & \text { Quadratic cost function }\end{array}$

\section{PRELIMINARY DEFINITIONS AND MODELING}

\subsection{Semiactive \& Regenerative Forcing Constraints}

Consider a controlled structure equipped with $m$ actuators and define $\mathbf{f}=\left\{f_{1} \ldots f_{m}\right\}^{T}$ as the vector of actuator forces. Define $\mathbf{v}=\left\{\begin{array}{lll}v_{1} & \ldots & v_{m}\end{array}\right\}^{T}$ as the corresponding vector of relative actuator velocities. For semiactive, "variable damping" systems, each $f_{i}$ is constrained to a range of velocity-proportional forces; i.e.,

$$
f_{i} \in\left[-c_{e i} v_{i}, 0\right]
$$

in which $c_{e i}$ is the maximum effective viscous damping of each device. (For a variable-orifice damper, $c_{e i}$ is the viscous damping of the device with the orifice in its "closed" position. For an electromechanical semiactive device, $c_{e i}$ is the forcevelocity ratio obtained by shorting the motor terminals.) Define the diagonal matrix $\mathbf{C}_{e}$ as

$$
\mathbf{C}_{e}=\operatorname{diag}\left\{c_{e 1} \ldots c_{c m}\right\}
$$

and define the vector of normalized forces $\mathbf{u}(t)$ as

$$
\mathbf{u}(t)=\mathbf{C}_{e}^{\mathrm{l} / 2} \mathbf{f}(t)
$$

Similarly, define the normalized actuation velocity vector $\mathbf{s}(t)$ as

$$
\mathbf{s}(t)=\mathbf{C}_{e}^{-1 / 2} \mathbf{v}(t)
$$

In terms of these normalized quantities, (1) is equivalent to

$$
u_{i}^{2}(t)+u_{i}(t) s_{i}(t) \leq 0
$$

It was shown in [8] that, like semiactive systems, RFA networks also exhibit velocity-dependent force limitations. Consider an $m$-device RFA network with force and velocity vectors $f$ and $\mathbf{v}$. (Note that any energy-storing elements, such as flywheels, may be incorporated into this system model as additional mechanical degrees of freedom.) The forces for RFA networks are limited by the single, global constraint

$$
\mathbf{f}^{T} \mathbf{C}_{e}^{-1} \mathbf{f}+\mathbf{f}^{T} \mathbf{v} \leq 0
$$

where $\mathbf{C}_{e}$ is the same diagonal matrix as discussed above for semiactive systems. In terms of $\mathbf{u}$ and $\mathbf{s ,}(6)$ is

$$
\mathbf{u}^{T}(t) \mathbf{u}(t)+\mathbf{u}^{T}(t) \mathbf{s}(t) \leq \mathbf{0}
$$

This quadratic equation has a useful graphical interpretation, as shown in Figure 2 for $m=2$. The feasible force region is a circle centered at a force vector equal to $-1 / 2 \mathbf{s}$. The boundary intersects with the origin of $\mathbf{u}$-space in such a way as to be orthogonal to $\mathbf{s}$. Also note that the dimensions of the region grow linearly with the magnitude of $\mathbf{s}$.

A comparison between constraints (5) and (7) makes clear the relationship between the two actuation systems in Fig. 1. For two systems constructed from the same hardware, the regenerative constraint is less restrictive than the semiactive constraint, because a single constraint is imposed on the entire network of actuators, whereas for semiactive systems the same constraint is imposed on each device separately. This is shown in Fig. 2, which illustrates that the semiactive feasible force region is rectangular, intersecting with the regenerative constraint boundary on the force axes.

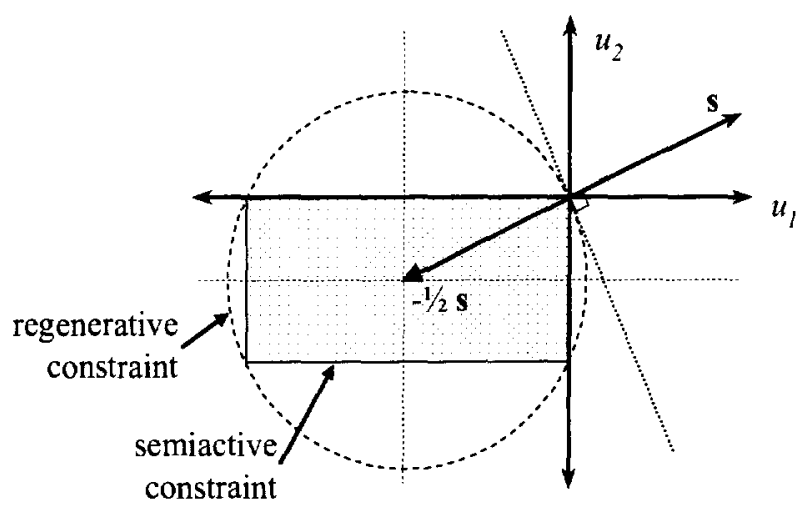

Figure 2: Feasible force regions for regenerative and semiactive two-actuator systems

Because semiactive and regenerative systems are "controllable-dissipation" systems, it is useful to view them as damping systems in which the damping coefficients are the control variables. This type of control approach is sometimes called "bilinear" [12], because the actuator force in Eq. (8) is linear in structural state, linear in control input, but nonlinear in 
both. Consider the relation of $\mathbf{u}(t)$ to $\mathbf{s}(t)$ through a controllable gain matrix $\mathbf{Z}(t)$, as

$$
\mathbf{u}(t)=-\mathbf{Z}(t) \mathbf{s}(t)
$$

Constraint (5) (for a semiactive system) or (7) (for a regenerative system) implies a constraint on the components of $\mathbf{Z}(t)$ which are feasible [13], expressed as

$$
\begin{aligned}
& \text { semiactive }: \begin{cases}\left|2 Z_{i j}(t)-1\right| \leq 1: & i=j \\
Z_{i j}(t)=0 & : i \neq j\end{cases} \\
& \text { regenerative : }\|2 \mathbf{Z}(t)-\mathbf{I}\|_{2} \leq 1
\end{aligned}
$$

The RFA network imposes a less-restrictive constraint, requiring only that the maximum singular value of $2 \mathrm{Z}-\mathrm{I}$ be less than unity. This wider domain of $\mathbf{Z}$ matrices allows for RFA networks to impose nonlocal and highly-asymmetric supplemental damping matrices on the structural systems.

The domain in Z-space characterized by Eq. (9) will be denoted $z$. Where it is necessary to differentiate between semiactive and regenerative domains, $Z_{S}$ and $Z_{R}$ will be used.

\subsection{Dynamic System Model}

Consider the standard second-order model for a linear, stochastically-excited, controlled structure, i.e.,

$$
\mathbf{M}_{s} \ddot{\mathbf{q}}(t)+\mathbf{C}_{s} \dot{\mathbf{q}}(t)+\mathbf{K}_{s} \mathbf{q}(t)=\mathbf{G a}(t)+\mathbf{N u}(t)
$$

External input vector $\mathbf{a}(t) \in \mathfrak{R}^{n_{a}}$ is considered to be a zero-mean, stationary Gaussian white noise process, and $\mathbf{G}$ is normalized such that $\mathbf{a}(t)$ has spectral intensity $\boldsymbol{\Phi}_{\boldsymbol{u}}=\mathbf{I}$. For this system, the standard state-space model is

$$
\dot{\mathbf{x}}(t)=\mathbf{A x}(t)+\mathbf{B}_{a} \mathbf{a}(t)+\mathbf{B}_{u} \mathbf{u}(t)
$$

where $\mathbf{x}(t) \in \Re^{n}$ is the system state, which is normalized such that $\mathbf{s}(t)$ is related to $\mathbf{x}(t)$ by

$$
\mathbf{s}(t)=\mathbf{B}_{u}^{T} \mathbf{x}(t)
$$

In the bilinear control formulation, $\mathbf{u}(t)$ is related to $\mathbf{x}(t)$ through

$$
\mathbf{u}(t)=-\mathbf{Z}(t) \mathbf{B}_{u}^{T} \mathbf{x}(t)
$$

for $\mathbf{Z}(t) \in \mathcal{Z}$. Bringing together Eqs. (11) and (13) gives

$$
\dot{\mathbf{x}}(t)=\left(\mathbf{A}-\mathbf{B}_{u} \mathbf{Z}(t) \mathbf{B}_{u}^{T}\right) \mathbf{x}(t)+\mathbf{B}_{a} \mathbf{a}(t) \quad, \quad \mathbf{Z}(t) \in Z
$$

\subsection{Quadratic Performance Measure}

Let the system outputs by which closed-loop performance is to be assessed be denoted $\mathbf{y}(t)$. It will be assumed that $\mathbf{y}(t)$ is a linear function of control and state variables; i.e.,

$$
\mathbf{y}(t)=\mathbf{C}_{y} \mathbf{x}(t)+\mathbf{D}_{y u} \mathbf{u}(t)=\left[\mathbf{C}_{y}-\mathbf{D}_{y u} \mathbf{Z}(t) \mathbf{B}_{u}^{T}\right] \mathbf{x}(t)
$$

The design goal is to find a controller such that the meansquare integral

$$
\phi(T)=\frac{1}{T} \int_{0}^{T} \mathbf{y}^{T}(t) \mathbf{y}(t) d t
$$

has favorable stochastic characteristics as $T \rightarrow \infty$. The performance measure used in this analysis will be the familiar Quadratic-Gaussian (QG) criterion, defined as

$$
J=\lim _{T \rightarrow \infty} E\{\phi(T)\}
$$

This performance criterion was chosen because it leads to the simplest analysis and controller derivation. However, as will be discussed later, analogous methods exist for other quadratic performance measures.

Throughout this paper, the assumption will be made that $\mathbf{D}_{y u}^{T} \mathbf{D}_{y u}>0$; i.e., that $\mathbf{y}(t)$ is defined such that $J$ penalizes all nonzero $\mathbf{u}(t)$.

\subsection{Optimal Constant-Z Design}

The simplest bilinear controller is a constant input; i.e., $\mathbf{Z}(t)=\mathbf{Z}_{0}, \forall t$, where $\mathbf{Z}_{0} \in Z$. In this case the control optimization problem reduces to a static output-feedback optimization over domain $z[13,14]$.

THEOREM 1: For the system (14), let $\mathbf{Z}_{0} \in Z$ be a constant matrix and assume $\mathbf{A}-\mathbf{B}_{u} \mathbf{Z}_{0} \mathbf{B}_{u}^{T}$ is stable. Then

$$
J=\operatorname{tr}\left\{\mathbf{B}_{a}^{T} \mathbf{P} \mathbf{B}_{a}\right\}
$$

where $\mathbf{P}$ obeys the Lyapunov equation

$$
\begin{aligned}
\mathbf{0} & =\left[\mathbf{A}-\mathbf{B}_{u} \mathbf{Z}_{0} \mathbf{B}_{u}^{T}\right]^{T} \mathbf{P}+\mathbf{P}\left[\mathbf{A}-\mathbf{B}_{u} \mathbf{Z}_{0} \mathbf{B}_{u}^{T}\right] \\
& +\left[\mathbf{C}_{y}-\mathbf{D}_{y u} \mathbf{Z}_{0} \mathbf{B}_{u}^{T}\right]^{T}\left[\mathbf{C}_{y}-\mathbf{D}_{y u} \mathbf{Z}_{0} \mathbf{B}_{u}^{T}\right]
\end{aligned}
$$

Furthermore,

$$
\frac{\partial J}{\partial \mathbf{Z}_{0}}=-2\left(\mathbf{B}_{u}^{T} \mathbf{P} \boldsymbol{\Phi} \mathbf{B}_{u}+\mathbf{D}_{y u}^{T}\left[\mathbf{C}_{y}-\mathbf{D}_{y u} \mathbf{Z}_{0} \mathbf{B}_{u}^{T}\right] \mathbf{\Phi} \mathbf{B}_{u}\right)
$$

where $\Phi$ obeys the Lyapunov equation

$$
\mathbf{0}=\left[\mathbf{A}-\mathbf{B}_{u} \mathbf{Z}_{0} \mathbf{B}_{u}^{T}\right] \boldsymbol{\Phi}+\boldsymbol{\Phi}\left[\mathbf{A}-\mathbf{B}_{u} \mathbf{Z}_{0} \mathbf{B}_{u}^{T}\right]^{T}+\mathbf{B}_{a} \mathbf{B}_{a}^{T}
$$

Proof: See [13].

Using (19)-(21), the optimum $\mathbf{Z}_{0}$ may be found through the method of steepest descent, and the corresponding $J$ can be evaluated from $\mathbf{Z}_{0}$ by (18). The optimal values will be denoted $\mathbf{Z}_{0}^{*}$ and $J_{0}^{*}$.

Denote the optimal pairs $\left\{\mathbf{Z}_{0}^{*}, J_{0}^{*}\right\}$ for domains $Z_{S}$ and $Z_{R}$ as $\left\{\mathbf{Z}_{0 S}^{*}, J_{0 S}^{*}\right\}$ and $\left\{\mathbf{Z}_{0 R}^{*}, J_{0 R}^{*}\right\}$, respectively. Then, note that since $\mathbf{Z}_{0 S}^{*}$ is diagonal (Due to constraint (9) on domain $Z_{S}$ ) and 
constant, it can be realized by linear viscous damping. Quantity $J_{0 S}^{*}$ is therefore the best performance achievable by viscous damping. Meanwhile, $\mathbf{Z}_{0 R}^{*}$ yields a $J_{0 R}^{*}$ which is guaranteed to be below $J_{0 S}^{*}$, because $Z_{S} \subset Z_{R}$. The margin of improvement $J_{0 S}^{*}-J_{0 R}^{*}$ afforded by the regenerative capability of the actuation network may be readily computed through Eq. (18), once $\mathbf{Z}_{0 S}^{*}$ and $\mathbf{Z}_{0 R}^{*}$ are known.

\section{STATE-FEEDBACK PGC'S}

\subsection{Controller Design}

This section presents a memoryless, full-state feedback law of the form $\mathbf{x}(t) \mapsto \mathbf{Z}(t)$, for which $J$ can be proved to possess an upper bound. Toward this end, the following lemma is instrumental.

LEMMA 2: For linear system (11), let $\mathbf{Z}_{0} \in Z$ be a constant matrix with $\mathbf{A}-\mathbf{B}_{u} \mathbf{Z}_{0} \mathbf{B}_{u}^{T}$ stable. Then in stationary response,

$$
\begin{aligned}
J & =\operatorname{tr}\left\{\mathbf{B}_{a}^{T} \mathbf{P} \mathbf{B}_{a}\right\}+E\left\|\mathbf{D}_{y u}(\mathbf{K} \mathbf{x}-\mathbf{u})\right\|_{2}^{2} \\
& -E\left\|\mathbf{D}_{y u}\left(\mathbf{K} \mathbf{x}+\mathbf{Z}_{0} \mathbf{B}_{u}^{T} \mathbf{x}\right)\right\|_{2}^{2}
\end{aligned}
$$

where $\mathbf{P}$ is defined as in (19) and $\mathbf{K}$ is defined as

$$
\mathbf{K}=-\left[\mathbf{D}_{y u}^{T} \mathbf{D}_{y u}\right]^{-1}\left[\mathbf{D}_{y u}^{T} \mathbf{C}_{y}+\mathbf{B}_{u}^{T} \mathbf{P}\right]
$$

In linear control theory, variants of this lemma can be used to characterize all controllers achieving a given level of performance [15]. For the bilinear systems considered here, it can be used for a similar purpose, as illustrated by the following theorem, which gives the desired performanceguaranteed control law.

THEOREM 3: For the control system in Eq. (14), let $\mathbf{Z}_{0}^{*} \in Z$ be the optimal constant $\mathbf{Z}$, and let $J_{0}^{*}$ be its performance. Let $\mathbf{P}$ and $\mathbf{K}$ be determined by Eqs. (19) and (23) with $\mathbf{Z}_{0}=\mathbf{Z}_{0}^{*}$. Then the following control law

$$
\mathbf{Z}(t)=\underset{\mathbf{Z} \in Z}{\arg \min }\left\|\mathbf{D}_{w u}\left(\mathbf{K}+\tilde{\mathbf{Z}} \mathbf{B}_{u}^{T}\right) \mathbf{x}(t)\right\|_{2}^{2}
$$

attains the performance level

$$
\begin{aligned}
J & =\operatorname{tr}\left\{\mathbf{B}_{a}^{T} \mathbf{P} \mathbf{B}_{a}\right\}+E\left\|\mathbf{D}_{y u}\left(\mathbf{K}+\mathbf{Z} \mathbf{B}_{u}^{T}\right) \mathbf{x}\right\|_{2}^{2} \\
& -E\left\|\mathbf{D}_{v u}\left(\mathbf{K}+\mathbf{Z}_{0}^{*} \mathbf{B}_{u}^{T}\right) \mathbf{x}\right\|_{2}^{2} \\
& \leq \operatorname{tr}\left\{\mathbf{B}_{a}^{T} \mathbf{P} \mathbf{B}_{u}\right\}=J_{0}
\end{aligned}
$$

Control law (24) guarantees to out-perform the case with $\mathbf{Z}(t)=\mathbf{Z}_{0}^{*}$. One can view this improvement in performance as arising from the instantaneous adjustment of $\mathbf{Z}(t)$ in response to information about the current state of the system, such that at any given time $t$, the disparity between the two norms in (22) is maximized. Because $J$ in Eq. (22) is referenced to a feasible control input, the difference in these norms can always be made negative.

\subsection{Resolving the clipping action}

The implementation of control law (24) requires, in general, the minimization of the quadratic quantity shown, over domain $Z$. As discussed in [10] and [11] it is more straight-forward to evaluate the minimizing $\mathbf{u}(t)=-\mathbf{Z}(t) \mathbf{B}_{n}^{T} \mathbf{x}(t)$ directly. For this purpose define the linear feedback signal $\mathbf{u}_{L}(t)$ as

$$
\mathbf{u}_{L}(t)=\mathbf{K x}(t)
$$

Then the bilinear controller in Eq. (24) is equivalent to the formulation of control force $u(t)$ as

$$
\mathbf{u}(t)=\underset{\text { ú feasible }}{\arg \min }\left\{\mid \mathbf{D}_{y u}\left(\tilde{\mathbf{u}}-\mathbf{u}_{L}(t)\right) \|_{2}^{2}\right\}
$$

where feasibility of $\tilde{\mathbf{u}}$ is determined from Eq. (5) for semiactive systems and Eq. (7) for RFA networks. Thus, $\mathbf{u}(t)$ tracks the linear feedback signal $\mathbf{u}_{L}(t)$ if it is feasible, and if not $\mathbf{u}_{L}(t)$ is "clipped" to the feasible region in such a way that the tracking error is instantaneously-optimized. Resolving this "clipping action" is a standard, straightforward optimization exercise. A general algorithm for resolving the clipping action for arbitrary $\mathbf{D}_{y u}$ is given in Ch. 6 of [18] and simple cases arising from assumptions on $\mathbf{D}_{y w}$ are briefly discussed in [10].

\section{OUTPUT FEEDBACK PGC'S}

Now, the case is considered in which the control system has access only to output data vector $\mathbf{z}(t) \in \mathfrak{R}^{n_{z}}$, which contains incomplete and possibly noise-corrupted information regarding the system state. We assume that $\mathbf{z}(t)$ may be expressed as

$$
\begin{aligned}
\mathbf{z}(t) & =\mathbf{C}_{z} \mathbf{x}(t)+\mathbf{D}_{z u} \mathbf{u}(t)+\mathbf{D}_{z n} \mathbf{n}(t) \\
& =\left[\mathbf{C}_{z}-\mathbf{D}_{z u} \mathbf{Z}(t) \mathbf{B}_{u}^{T}\right] \mathbf{x}(t)+\mathbf{D}_{z n} \mathbf{n}(t)
\end{aligned}
$$

where $\mathbf{n}(t) \in \mathfrak{R}^{n_{n}}$ is the sensor noise, modeled as a stationary Gaussian white noise vector process. $\mathbf{D}_{z n}$ is normalized such that $\mathbf{n}(t)$ has spectral intensity $\boldsymbol{\Phi}_{n}=\mathbf{I}$. Furthermore, the following two assumptions are made:

1) Correlation between $\mathbf{n}(t)$ and $\mathbf{a}(t)$ is not considered here, although the incorporation of this correlation does not substantively alter the ideas.

2) In addition to any other noisy feedback signals, $z(t)$ includes $\mathbf{s}(t)$ and $\mathbf{u}(t)$ with zero noise intensity; i.e., the rows of $\mathbf{D}_{z n}$ corresponding to these signals contain all zeros.

For such a scenaro, we seek a feedback law of the form $\mathbf{z}[0, t] \mapsto \mathbf{Z}(t)$, for which $J$ can be shown to possess an upper 
bound. Here, this is accomplished in a two-stage manner analogous to LQG control, by first designing an observer, and then designing a controller which operates on the observed system states.

For this discussion, it will be assumed for convenience of presentation that the system states are partitioned as

$$
\mathbf{x}=\left[\begin{array}{l}
\mathbf{x}_{1} \\
\mathbf{x}_{2}
\end{array}\right], \quad \mathbf{B}_{u}=\left[\begin{array}{c}
\mathbf{B}_{u 1} \\
\mathbf{0}
\end{array}\right]
$$

where $\mathbf{B}_{u 1}$ is square and nonsingular. (This assumption can be made without loss of generality, so long as $\mathbf{B}_{u}$ has full column rank.) Because $\mathbf{s}(t)$ is known exactly, $\mathbf{x}_{1}(t)=\mathbf{B}_{u 1}^{-T} \mathbf{s}(t)$ is as well, and it is only necessary to estimate $\mathbf{x}_{2}(t)$.

It should be noted that observers for similar problems have been investigated in the context of semiactive vehicle suspensions [16, 17], which employed asymptoticallyconvergent observers. However, these observers require very specific restrictions to be placed on the performance measure and the sensor locations. The present analysis applies to general circumstances.

\subsection{Observer Design}

In order for the controller and observer designs to be independent as in LQG control, the control problem must be framed such that it exhibits certainty-equivalence. In the present context, this requirement imposes the condition that the estimated state vector should be unbiased, with respect to its error residual. As will be shown below, this requires that the observer be designed in a specific way.

From the classical theory of linear, time-invariant stochastic observers, if $\mathbf{u}[0, t]$ is known precisely then $\mathbf{x}(t)$ can be estimated as from data $\mathbf{z}[0, t]$ through the observer

$$
\dot{\tilde{\mathbf{x}}}(t)=\mathbf{A} \tilde{\mathbf{x}}(t)+\mathbf{B}_{u} \mathbf{u}(t)+\mathbf{F}\left(\mathbf{z}(t)-\mathbf{C}_{z} \tilde{\mathbf{x}}(t)-\mathbf{D}_{z u} \mathbf{u}(t)\right)
$$

where $\mathbf{F}$ is any matrix such that $\mathbf{A}-\mathbf{F} \mathbf{C}_{z}$ is stable. Although the resultant vector $\tilde{\mathbf{x}}_{2}(t)$ could be used as an estimate for $\mathbf{x}_{2}(t)$, its error residual $\mathbf{r}_{2}(t)=\mathbf{x}_{2}(t)-\tilde{\mathbf{x}}_{2}(t)$ is in general biased with respect to $\tilde{\mathbf{x}}_{2}(t)$ and $\mathbf{x}_{1}(t)$. Note that here, this bias cannot be removed through optimization (i.e., by designating $\mathbf{F}$ as the optimal Kalman gain). Because $\mathbf{u}$ and $\mathbf{s}$ are assumed to be known with certainty, $\mathbf{D}_{z n}$ is singular and as such, the resultant optimization problem is ill-posed. However, so long as $\mathbf{F}$ is chosen such that $\mathbf{A}-\mathbf{F} \mathbf{C}_{z}$ is stable, this bias may be "subtracted off" to obtain an unbiased estimate $\hat{\mathbf{x}}_{2}(t)$. Lemma 4 below illustrates how to do this.

LEMMA 4: In stationary response, the probability density $p\left(\mathbf{x}_{2}(t) \mid \tilde{\mathbf{x}}(t), \mathbf{x}_{1}(t)\right)$ is Gaussian, with mean $\hat{\mathbf{x}}_{2}(t)$ equal to

$$
\hat{\mathbf{x}}_{2}(t)=\tilde{\mathbf{x}}_{2}(t)+\left[\begin{array}{ll}
\tilde{\mathbf{S}}_{13}^{T} & \tilde{\mathbf{S}}_{23}^{T}
\end{array}\right]\left[\begin{array}{ll}
\tilde{\mathbf{S}}_{11} & \tilde{\mathbf{S}}_{12} \\
\tilde{\mathbf{S}}_{12}^{T} & \tilde{\mathbf{S}}_{22}
\end{array}\right]^{-1}\left[\begin{array}{c}
\tilde{\mathbf{x}}_{(t)} \\
\mathbf{x}_{1}(t)-\tilde{\mathbf{x}}_{1}(t)
\end{array}\right]
$$

and with time-invariant covariance matrix $\boldsymbol{S}_{r 2}$ equal to

$$
\mathbf{S}_{r 2}=\tilde{\mathbf{S}}_{33}-\left[\begin{array}{ll}
\tilde{\mathbf{S}}_{13}^{T} & \tilde{\mathbf{S}}_{23}^{T}
\end{array}\right]\left[\begin{array}{ll}
\tilde{\mathbf{S}}_{11} & \tilde{\mathbf{S}}_{12} \\
\tilde{\mathbf{S}}_{12}^{T} & \tilde{\mathbf{S}}_{22}
\end{array}\right]^{-1}\left[\begin{array}{c}
\tilde{\mathbf{S}}_{13} \\
\tilde{\mathbf{S}}_{23}
\end{array}\right]
$$

where the $\tilde{\mathbf{S}}_{i j}$ matrices are computed from the algebraic Lyapunov equation

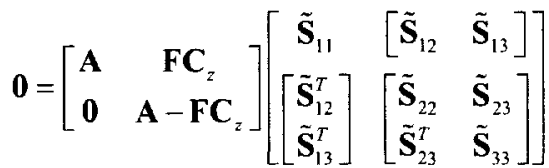

$$
\begin{aligned}
& +\left[\begin{array}{cc}
\tilde{\mathbf{S}}_{11} & {\left[\begin{array}{ll}
\tilde{\mathbf{S}}_{12} & \tilde{\mathbf{S}}_{13}
\end{array}\right]} \\
{\left[\begin{array}{c}
\tilde{\mathbf{S}}_{12}^{T} \\
\tilde{\mathbf{S}}_{13}^{T}
\end{array}\right]\left[\begin{array}{ll}
\tilde{\mathbf{S}}_{22} & \tilde{\mathbf{S}}_{23} \\
\tilde{\mathbf{S}}_{23}^{T} & \tilde{\mathbf{S}}_{33}
\end{array}\right]}
\end{array}\right]\left[\begin{array}{cc}
\mathbf{A} & \mathbf{F C} z \\
\mathbf{0} & \mathbf{A}-\mathbf{F C}
\end{array}\right]^{T}
\end{aligned}
$$

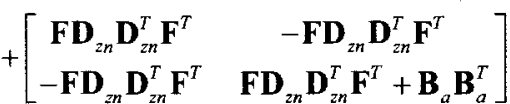

Lemma 4 applies to observers with any stabilizing $\mathbf{F}$ in Eq.(30). Thus, $\mathbf{F}$ can be chosen independently, to satisfy other constraints that may exist for control system design. One simple approach is to choose $\mathbf{F}$ as the Kalman gain corresponding to a problem with more sensor noise than actually exists. To do this, consider the introduction of additional, fictitious noise into Eq. (28) for $\mathbf{z}(t)$; i.e.,

$$
\mathbf{z}^{f i c}(t)=\mathbf{C}_{z} \mathbf{x}(t)+\mathbf{D}_{z u} \mathbf{u}(t)+\mathbf{D}_{z n} \mathbf{n}(t)+\mathbf{D}_{z n}^{f i c} \mathbf{n}^{f i c}(t)
$$

where the "fic" superscript is to remind us that the new noise term is a fictitious construct used only to design $\mathbf{F}$. As with the actual sensor noise, $\mathbf{n}^{\text {fic }}$ is assumed to be unit-intensity whitenoise, and $\mathbf{D}_{z n}^{\hat{f i c}}$ determines the correlation and intensity of this noise. The optimal (i.e., Kalman) $\mathbf{F}$ for this case is then

$$
\mathbf{F}=\mathbf{S}^{f i c} \mathbf{C}_{z}^{T}\left[\mathbf{D}_{z n} \mathbf{D}_{z n}^{T}+\mathbf{D}_{z n}^{f i c} \mathbf{D}_{z n}^{f i c}\right]^{-1}
$$

where $\mathbf{S}^{\text {fic }}$ obeys the algebraic Riccati equation

$$
\begin{aligned}
\mathbf{0} & =\mathbf{B}_{a} \mathbf{B}_{a}^{T}+\mathbf{A} \mathbf{S}^{f i c}+\mathbf{S}^{f i c} \mathbf{A}^{T} \\
& -\mathbf{S}^{f i c} \mathbf{C}_{z}^{T}\left[\mathbf{D}_{z n} \mathbf{D}_{z n}^{T}+\mathbf{D}_{2 n}^{f i c} \mathbf{D}_{2 n}^{f i c}\right]^{-1} \mathbf{C}_{z} \mathbf{S}^{f i c}
\end{aligned}
$$

It is well-known that as $\mathbf{D}_{z n}^{f i c} \mathbf{D}_{z n}^{f i c}$ is increased, the bandwidth of the resultant observer tends to decrease, thus allowing for indirect controller bandwidth design.

\subsection{Controller Design}

Having constructed an unbiased observer, the development of a control algorithm analogous to that of Theorem 3 follows directly.

THEOREM 5: For the control system (14) with measurement outputs (28), let $\mathbf{Z}_{0}^{*}, J_{0}^{*}, \mathbf{P}$ and $\mathbf{K}$ be defined as in Theorem 3. 
Assume that $\mathbf{u}(t)$ and $\mathbf{s}(t)$ in (12) and (13) are contained in $\mathbf{z}(t)$ and are measured without noise. Let the system states be partitioned as in (29), and let $\hat{\mathbf{x}}_{2}(t)$ be related to $\tilde{\mathbf{x}}(t)$ through (31). Then the control law

$$
\mathbf{Z}(t)=\underset{\tilde{\mathbf{Z}} \in Z}{\arg \min }\left\{\left\|\mathbf{D}_{y u}\left(\mathbf{K}\left[\begin{array}{c}
\mathbf{B}_{u 1}^{-T} \mathbf{s}(t) \\
\hat{\mathbf{x}}_{2}(t)
\end{array}\right]+\tilde{\mathbf{Z}} \mathbf{s}(t)\right)\right\|_{2}^{2}\right\}
$$

attains the performance level

$$
\begin{aligned}
J & =\operatorname{tr}\left\{\mathbf{B}_{a}^{T} \mathbf{P} \mathbf{B}_{a}\right\}+E\left\|\mathbf{D}_{y u}\left(\mathbf{K}\left[\begin{array}{c}
\mathbf{B}_{u 1}^{-T} \mathbf{s} \\
\hat{\mathbf{x}}_{2}
\end{array}\right]+\mathbf{Z s}\right)\right\|_{2}^{2} \\
& -E\left\|\mathbf{D}_{y u}\left(\mathbf{K}\left[\begin{array}{c}
\mathbf{B}_{u 1}^{-T} \mathbf{s} \\
\hat{\mathbf{x}}_{2}
\end{array}\right]+\mathbf{Z}_{0}^{*} \mathbf{s}\right)\right\|_{2}^{2} \\
& \leq \operatorname{tr}\left\{\mathbf{B}_{a}^{T} \mathbf{P} \mathbf{B}_{a}\right\}=J_{0}^{*}
\end{aligned}
$$

The mathematical structure of the control law (37) is the same as the full-state PGC from Theorem 3 except that $\hat{\mathbf{x}}_{2}$ is used here in place of $\mathbf{x}_{2}$, which was there assumed to be known. Thus, the output-feedback PGC above exhibits certaintyequivalence.

The implementation of control law (37) can be performed in a similar way to the output feedback case by evaluating the minimizing $\mathbf{u}(t)=\mathbf{Z}(t) \mathbf{s}(t)$ directly.

\section{EXAMPLE}

Consider the two-story shear building example shown in Fig. 3. Each 80 -ton floor is $20 \mathrm{~m}$ on all sides, with a radius of gyration of $8.61 \mathrm{~m}$. The flexural story stiffnesses in both lateral directions is $9 \times 10^{4} \mathrm{kN} / \mathrm{m}$, and the torsional stiffness of a story is $7.08 \times 10^{9} \mathrm{Nm} / \mathrm{rad}$. The eccentricities between the mass and stiffness centers in both floors in $x$ - and $y$-directions are 0.125 and 0.075 respectively. Modal damping equal to $2 \%$ is assumed for all modes. The lowest three natural frequencies are 17.6, 20.7 , and $25.0 \mathrm{rad} / \mathrm{s}$.

The building model is subjected to earthquake excitation in the $x$ and $y$ directions. This excitation is modeled as two independent, stationary stochastic processes, with second-order Kanai-Tajimi spectra. The states for the earthquake model are appended to the structural states to form a 16-state earthquakestructure simulation model.

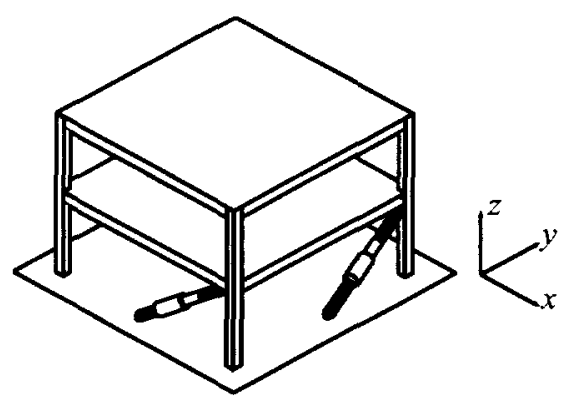

Figure 3: Example structure
In $[10,11]$, this structural model is used to demonstrate at length the efficacy of a number of PGC strategies. The discussion here reflects only some of those observations. The control actuators are placed between the ground and the first floor. For the purpose of demonstration, it is assumed that the first floor of the structure is a large open area (such as a lobby) with windows on two adjacent sides and that consequently, for aesthetic reasons, actuators may only be placed in plane with the two remaining sides of the lower bay as shown. Both actuators are modeled identically, with $c_{e i}=10^{7} \mathrm{~kg} / \mathrm{s}$. Electromechanical device realizations similar to those in Fig. 1 are assumed. Both semiactive and regenerative realizations will be considered in this example. The RFA network will not be assumed to have an energy-storage element included, as it was found that for this example, the ability of the RFA network to transmit energy between the different degrees of freedom was the more critical asset for response reduction.

The output performance variables are taken as the structural drifts at the corner furthest from the center of stiffness, and the absolute accelerations at the center of mass of both floors in both $\mathrm{x}$ - and $\mathrm{y}$ - directions. Thus,

$$
\begin{aligned}
& \mathbf{y}(t)= {\left[\begin{array}{llll}
w_{d}\left[d_{1 x}(t)\right. & d_{1 y}(t) & d_{2 x}(t) & d_{1 y}(t)
\end{array}\right] } \\
& w_{a}\left[\begin{array}{llll}
a_{1 x}(t) & a_{1 y}(t) & a_{2 x}(t) & a_{1 y}(t)
\end{array}\right]^{T}
\end{aligned}
$$

where quantities $w_{d}$ and $w_{a}$ are design weights which will be used to adjust the relative tradeoff between drifts and accelerations for the controller design.

For each performance ratio, four control cases are presented here: PGC's with semiactive and regenerative actuation, and Constant- $\mathbf{Z}$ designs with semiactive and regenerative constraints. These will be refferred to as PGS, PGR, CZS, and CZR cases. The performance of these control systems will be measured relative to the CZS case, i.e., the marginal improvement afforded by the control system over optimal linear viscous damping. The reasoning is that viscous dampers are much simpler and cheaper than controllable devices, and the improvement in performance afforded by these devices should justify the increased cost. Thus, $J_{\text {Czs }}$ is useful as a benchmark by which all other $J \mathrm{~s}$ can be judged.

Both state- and output-feedback controllers are considered in this example. For the output-feedback controllers, sensor feedback vector $\mathbf{z}(t)$ was taken to be only the actuator velocities and control forces, which are assumed to be measured in the absence of noise. Thus, $\mathbf{D}_{z n}=\mathbf{0}$.

Gain $\mathbf{F}$ must be designed for the output-feedback observer in Eq.(30). This will be done in the manner described following Lemma 4, through the design of a Kalman filter with fictitious sensor noise matrix $\mathbf{D}_{z n}^{f i c}=d_{z n}^{f i c} \mathbf{I}_{2}$, where scalar $d_{z n}^{\text {fic }}$ can be designed to achieve acceptable transient characteristics for the filter. Here, two designs are considered, with successively-more restrictive limitations on controller bandwidth. These designs correspond to $d_{z n}^{\text {fic }}$ values of 
$0.5 \times 10^{-4}$ and $3.75 \times 10^{-3}$, and result in observers with maximum pole moduli of 100 and $10 \mathrm{~Hz}$, respectively.

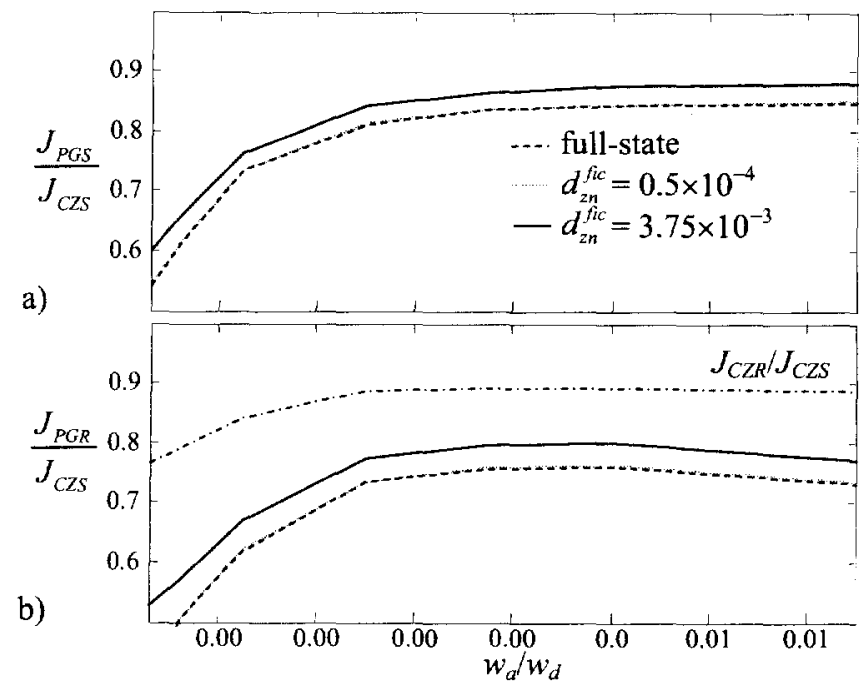

Figure 4: Performances of semiactive (a) and regenerative (b) PGC's for example 1

Fig. 4 shows $J$ for semiactive and regenerative PGC's, evaluated over a range of design weight ratios $w_{a} / w_{d}$. Data for both state-feedback and output-feedback PGC's are shown . For the state-feedback case, is is clear that over the entire range of $w_{d} / w_{d}$ values, the controller designs consistently out-perform optimal viscous damping (the CZS case) for both semiactive and regnerative controllers. Also as predicted, the regenerative controllers consistently yield an improvement in performance which is bounded by the ratio $J_{\mathrm{CZR}} / J_{\mathrm{CZS}}$. Thus, regenerative systems out-perform optimal viscous damping by a computable margin.

Output-feedback controllers are given for the two observer designs. It is clear that, as $d_{z n}^{\text {fic }}$ is made to decrease to zero, the performances of the various controller designs become close to that of the full-state design, and that for finite $d_{z n}^{\text {ic }}$, the departure from the performance of the full-state PGC is relatively consistent over the performance metrics considered. No matter how sub-optimal the observer may be (i.e., no matter how large $d_{z n}^{f i c}$ is made), the resultant output-feedback PGC will always adhere to the same guaranteed performance margins as the state-feedback controller. However, improvement in performance beyond this guaranteed margin typically diminishes with tighter restrictions on observer bandwidth.

\section{EXTENSIONS TO OTHER CONTROL PROBLEMS}

There are many other control problems for which variants of PGC methods exist. Here, we briefly discuss three such extensions. However, in the interest of brevity, discussion of each of these approaches is limited to qualitative observations.

\subsection{Noisy Velocity and Force Measurements}

It is straight-forward to apply these methods to problems for which the noise assumptions for the system outputs $\mathbf{z}$ are different from those made here. One example is the case where both the actuator velocities $\mathbf{s}$ and forces $\mathbf{u}$ are assumed to be measured in the presence of noise, but where the bilinear control input $\mathbf{Z}$ can be assumed to be known with certainty.

It turns out that the development PGC's for this case follow in an even more straight-forward manner than the case considered in this paper, and are presented in detail in [10]. The resultant control law is similar to (37), but the minimization is performed on two norms instead of just one. The first of these is analogous to the norm in (37), and its minimization reflects the effort of the controller to reduce the response magnitude beyond the constant $\mathbf{Z}$ case, assuming the state estimate to be accurate. The second norm in the minimization is a function of $\mathbf{Z}$ and the time-varying error covariance of the system state estimate. The presence of this term reflects the effort of the controller to adjust $\mathbf{Z}$ so as to obtain a better state estimate. Thus, by minimization of a summation of both these norms, the controller is required to balance the dual roles of achieving better control and better observation. Such dual control objectives are common in nonlinear and adaptive control and arise in many problems, such as this one, where certainty-equivalence cannot be assumed.

A more challenging problem concerns the case where the control forces $\mathbf{u}$ are measured in the absence of noise and are prescribed directly by the control system, but where uncertainty exists for $\mathbf{s}$. This case is problematic because it is impossible for the controller to impose constraints (5) or (7) on $\mathbf{u}$ if $\mathbf{s}$ is uncertain. Preliminary research in this are shows that PGC's exist for very specific circumstances, but a general synthesis remains elusive.

\subsection{Problems Related to Risk-Sensitive Control}

For $\not(T)$ as defined in (16), the risk-sensitive peformance measure exponentially penalizes large values of $\phi(T)$, thus assigning disproportional preference to controllers which produce distributions for $\phi(T)$ with smaller mass in the tails. Specifically, our interest is in the limiting case as $T \rightarrow \infty$, which can be expressed as

$$
H(\gamma)=\lim _{T \rightarrow \infty} \frac{1}{\gamma} \ln \{E\{\exp [\gamma \phi(T)]\}\}
$$

where $\gamma>0$ is a design parameter which reflects the degree of risk-averseness for the performance measure.

Extensive research has been conducted on the optimization of feedback controllers for minimal $H(\gamma)$. It has been shown that this control problem exhibits a kind of certaintyequivalence similar to LQG control (which, in fact, corresponds to the limiting case as $\gamma \rightarrow 0$ ). Connections exist to the $H_{\infty}$ optimal control problem, which may be interpreted as the derivation of the risk-sensitive controller yielding the largest admissible $\gamma$. 
Semiactive and regenerative PGC's can be designed which yield a guaranteed risk-sensitive and $H_{\infty}$ margins. This is done in [18], for the $H_{\infty}$ case.

\subsection{Multi-Objective Control Problems}

The controllers presented in this paper have been designed to guarantee a level of scalar performance. However, an interesting question concerns whether the method can be extended so as to guarantee satisfaction of a set of quadratic performance measures. It is straight-forward to show that this can be done through proper use of Linear Matrix Inequalities (LMI's) [19].

\section{CONCLUSIONS}

This paper has presented a systematic approach to the design of nonlinear control laws for a general class of controllably-dissipative systems involving semiactive and/or regenerative actuators. The method's appeal lies in its guaranteed performance margins for closed-loop response, which sets it apart from many other control design approaches for these systems. For systems with complex dynamics, this may prove a useful design tool. Although the approach was presented here in the context of Quadratic-Gaussian stochastic control, the strategy appears to be quite general and applicable in several other contexts.

\section{ACKNOWLEDGMENTS}

This research was supported by NSF SGER grant CMS0505887. This support is gratefully acknowledged.

The authors would like to thank Professor James L. Beck for helpful comments on the manuscript.

\section{REFERENCES}

[1]. Hrovat, D., Survey of Advanced Suspension

Developments and Related Optimal Control Applications. Automatica, 1997. 33(10): p. 1781-1817.

[2]. Chu, S.Y., T.T. Soong, and A.M. Reinhorn, Active, Hybrid, and Semi-active Control - A Design and Implementation Handbook. John Wiley \& Sons, Ltd.: 2005, Chichester and New York.

[3]. Wang, K.W., Structural Vibration Control Utilizing Piezoelectric Networks, in The Mechanical Systems Design Handbook: Modeling, Measurement, and Control, H.a. Nwokah, Editor. 2001, CRC Press. p. Chapter 15.

[4]. Karnopp, D.C., Permanent Magnet Linear Motors Used as Variable Mechanical Dampers for Vehicle Suspensions. Vehicle System Dynamics, 1989. 18: p. 187-200.

[5]. Scruggs, J.T. and W.D. Iwan, Control of a Civil Structure Using an Electric Machine with Semiactive Capability. ASCE Journal of Structural Engineering, 2003. 129(7): p. 951-959. [6]. Jolly, M.R. and D.L. Margolis, Regenerative Systems for Vibration Control. Journal of Vibration and Acoustics, 1997. 119(April): p. 208-215.

[7]. Nerves, A.C. and R. Krishnan, A Strategy for Active Control of Tall Civil Structures Using Regenerative Electric
Actuators, in Proc., Engineering Mechanics Conference. 1996: Fort Lauderdale, FL. p. 503-506.

[8]. Scruggs, J.T. and W.D. Iwan, Structural Control with Regenerative Force Actuation Networks. Journal of Structural Control and Health Monitoring, 2005. 12(1): p. 24-45.

[9]. Tseng, H.E. and J.K. Hedrick, Semi-Active Control Laws Optimal and Sub-Optimal. Vehicle System Dynamics, 1994. 23: p. 545-69.

[10]. Scruggs, J.T., A.A. Taflanidis, and W.D. Iwan, Nonlinear Stochastic Controllers for Semiactive and Regenerative Systems with Guaranteed Mean-Square Performance. Part 1: State Feedback Control. Journal of Structural Control \& Health Monitoring (submitted), 2005.

[11]. Scruggs, J.T., A.A. Taflanidis, and W.D. Iwan, Nonlinear Stochastic Controllers for Semiactive and Regenerative Systems with Guaranteed Mean-Square Performance. Part 2: Output Feedback Control. Journal of Structural Control \& Health Monitoring (submitted), 2005.

[12]. Mohler, R.R., Bilinear Control Processes. Mathematics in Science and Engineering, ed. R. Bellman. Vol. 106. Academic Press: 1973, New York.

[13]. Scruggs, J.T. and W.D. Iwan, Optimal Non-Local and Asymmetric Structural Damping Using Regenerative Force Actuation Networks. Journal of Engineering Mechanics (accepted), 2005.

[14]. Scruggs, J.T. and W.D. Iwan. Dissipative Capabilities of Regenerative Force Actuators. in International Symposium on Network and Center-Based Research for Smart Structures Technologies and Earthquake Engineering. 2004. Osaka. [15]. Doyle, J., K. Glover, P. Khargonekar, and B. Francis, State-Space Solutions to Standard H2 and H-Infinity Control Problems. IEEE Transactions on Automatic Control, 1988. 34(8): p. 831-847.

[16]. Hedrick, J., R. Rajamani, and K. Yi, Observer Design for Electronic Suspension Applications. Vehicle System Dynamics, 1994. 23: p. 413-440.

[17]. Yi, K. and B. Song, Observer Design for Semi-Active Suspension Control. Vehicle System Dynamics, 1999. 32: p. 129-148.

[18]. Scruggs, J.T., Structural Control Using Regenerative Force Actuation Networks. 2004, Ph.D. dissertation, Div. Engr. \& Appl. Sci., Caltech.

[19]. Scruggs, J.T., Multi-Objective, Performance-Guaranteed Control of Semiactive and Regenerative Systems. In progress, 2006. 